\title{
Place et fonctions de la langue française en Tunisie
}

Noureddine Sraîeb

\section{(2) OpenEdition \\ 1 Journals}

Édition électronique

URL : https://journals.openedition.org/dhfles/2927

DOI : $10.4000 /$ dhfles. 2927

ISSN : 2221-4038

Éditeur

Société Internationale pour l'Histoire du Français Langue Étrangère ou Seconde

Édition imprimée

Date de publication : 1 décembre 2000

Pagination : 34-45

ISSN : 0992-7654

\section{Référence électronique}

Noureddine Sraîeb, «Place et fonctions de la langue française en Tunisie », Documents pour l'histoire du français langue étrangère ou seconde [En ligne], 25 | 2000, mis en ligne le 04 octobre 2014, consulté le 27 mai 2021. URL : http://journals.openedition.org/dhfles/2927; DOl : https://doi.org/10.4000/dhfles. 2927

Ce document a été généré automatiquement le 27 mai 2021.

(c) SIHFLES 


\title{
Place et fonctions de la langue française en Tunisie
}

\author{
Noureddine Sraîeb
}

1 C'est à partir du deuxième tiers du XIX siècle que la langue française commença à être utilisée en Tunisie. Elle fut introduite par deux canaux différents : les écoles congréganistes et le pouvoir politique tunisien. Nous aurons donc à examiner les raisons qui ont favorisé cette introduction de la langue française et les objectifs qui lui étaient assignés.

2 Cette situation fut maintenue voire renforcée par l'instauration du régime du Protectorat. Quels furent donc les effets de cette nouvelle conjoncture politique sur le statut de la langue française ? Quels étaient les enjeux qu'elle représentait pour le pouvoir colonial, le parti colon et les nationalistes tunisiens? Quel fut l'avenir de la langue française dans la Tunisie indépendante?

\section{Les conditions d'introduction de la langue française}

Dès 1705 la Tunisie est gouvernée par la dynastie husseïnite, d'origine turque. Cette dynastie, malgré ses origines, s'était très vite intégrée à la population autochtone et des mariages furent conclus entre des membres de la famille beylicale et de notables tunisiens. Cela fit dire à des historiens de la Tunisie contemporaine qu'il s'agissait d'une dynastie nationale. Malgré des liens de vassalité plus ou moins ténus entre la Régence de Tunis et la Sublime Porte, des rapports très étroits étaient maintenus grâce aux missions diplomatiques et aux séjours prolongés ou brefs d'intellectuels tunisiens, cheikhs issus de l'Université de la Zitouna de Tunis, qui finirent par avoir des relations étroites avec des ulémas et hommes d'État ottomans. Aussi les informations pouvaientelles circuler facilement au point que ces ulémas seront des médiateurs culturels et politiques. Ce fut le cas du cheikh Mahmud Qabadu, ce fut également le cas du cheikh Ahmad Ibn Abi Diyaf, pour ne citer que ces deux personnalités.

D'autre part, la Tunisie disposait d'un État centralisé qui étendait son pouvoir sur tout le Royaume divisé en régions à la tête desquelles des gouverneurs ou caïds 
représentaient le pouvoir central, ce qui n'était pas le cas du Maroc où le pays était divisé entre le bilad al-mahzin et le bilad as-siba, comme on les appelait à l'époque et où les contacts avec la Sublime Porte étaient pratiquement inexistants, le Maroc étant resté replié sur lui-même ou n'ayant de contacts qu'avec l'Europe. Même dans ce cas, les ulémas marocains, quoique séduits par les réalisations européennes, restaient hostiles à toute idée d'emprunt à l'Europe.

Quant à l'Algérie, elle était, alors, une colonie française et les rapports de la population algérienne avec la Sublime Porte étaient inexistants. C'est ce qui l'éloigna de tout le mouvement de réformes qui touchait une bonne partie du monde musulman dont, notamment, la Turquie, l'Egypte et la Tunisie en ce deuxième tiers du XIXe siècle.

Ce préambule historique me semblait nécessaire pour marquer les différences qui existaient entre les trois pays du Maghreb. Ces situations avaient des répercussions sur les politiques scolaires adoptées dans chacun des pays. En effet, l'adoption du français fut imposée, en Algérie, dès le début de l'occupation du pays par la France. Au Maroc, l'enseignement était du ressort des ulémas de la Qarawiyin de Fès et il ne commença à subir des réformes qu'à partir des années 1934. La situation de la Tunisie était, comme nous le verrons, bien différente.

\section{L'enseignement congréganiste}

7 L'existence de communautés européennes en Tunisie, comme au Maroc d'ailleurs, avant l'occupation française, exigeait la création d'écoles pour les enfants européens au besoin desquels l'enseignement tunisien, tel qu'il était conçu, ne pouvait pas répondre. Des écoles furent, donc, créées à partir de 1831.

8 A cette date Pompeo Sulema, émigré politique livournais créait avec sa sœur Esther une école italienne, tandis que d'autres écoles, maltaise, britannique et israélite étaient ouvertes à la même époque. L'abbé François Bourgade, nommé en 1841, aumônier de la Chapelle St-Louis à Carthage, chapelain du Consulat de France, décidait de créer une école, avec la collaboration de P. Sulema et la participation financière et matérielle des commerçants français qui lui cédèrent une maison. De quelques dizaines en 1841, les effectifs furent de 300 et en 1845 l'école prit le nom de Collège Saint-Louis. « On y pratiquait toutes les disciplines de l'enseignement primaire et secondaire : littérature française, arithmétique, sciences naturelles, italien, chimie, physique, dessin linéaire $»^{1}$.

Ce fut là le début de l'introduction de l'enseignement européen dans la Régence. Cet enseignement, signalons-le, concernait aussi bien les garçons que les filles depuis que, dès octobre 1840, la sœur Emilie de Vialar créait des écoles pour jeunes filles. Ces écoles ne seront pas limitées à Hinis puisque d'autres écoles seront créées, en 1843 à Sousse, en 1852 à Sfax, en 1855 à La Goulette et en 1879 à Djerba.

Notons, cependant, que si les écoles de garçons comptaient « à peu près 80 indigènes tant Israélites qu'arabes ", les écoles de filles ne recevaient pas encore de jeunes filles musulmanes.

11 Les frères de la Doctrine Chrétienne devaient, à leur tour, ouvrir des écoles sous la direction de Mgr Sutter, Vicaire apostolique et Administrateur temporel des biens de la mission. Il voulait contre-balancer l'œuvre de l'abbé F. Bourgade. Soupçonnés de vouloir promouvoir la langue italienne au détriment de la langue française, les aides qu'ils réclamaient au gouvernement français étaient soit réduites soit supprimées. 
L'hostilité qu'il y avait entre Mgr Sutter et le Cardinal Lavigerie nous intéresse dans la mesure où le " conflit linguistique " franco-italien était l'expression des velléités politiques italo-françaises, chacune des deux puissances voulant établir son hégémonie sur le pays convoité. Cela apparaît, notamment, à travers une Note sur la situation des écoles chrétiennes en Tunisie, en date du 14 août $1880^{2}$. C'est, en effet, à la suite d'une demande de subvention adressée par Mgr Sutter au ministre des Affaires étrangères à Paris que certaines considérations politiques sont évoquées. Le rédacteur de la Note rejette systématiquement la requête de Mgr Sutter sous prétexte que " Mgr Sutter, dont la situation n'est pas aussi mauvaise que sa lettre le ferait croire, ne dit pas à quel usage il affecterait les économies qu'il lui serait permis de réaliser ; en tout cas ", ajoute la Note, "les sacrifices que le Gouvernement de la République s'impose pour le développement de nos écoles doivent profiter exclusivement à celles-ci et ne peuvent contribuer à grossir par une voie indirecte le budget particulier de l'Évêché ».

On invoque ainsi le principe de la séparation de l'Église et de l'État pour repousser la demande de Mgr Sutter mais on demande, cependant, « de porter toutes ces ressources disponibles aux écoles des frères et des sœurs de Tunis » rappelant tout l'intérêt qu'il y a à développer l'instruction primaire dans la Régence où " le Gouvernement italien consacre à subventionner des écoles de sa nationalité une somme de 11500 francs par an ». L'auteur signale, an passage, "le dévouement à la France et les sentiments patriotiques des frères et des sœurs dont l'action se trouve entravée par le caractère même de la mission catholique qui est italienne, en sorte que l'instruction religieuse se fiât en italien ». Il recommande, donc, d'accorder la subvention aux écoles des sœurs et des frères et de "stipuler expressément, [...] que cette subvention devra mettre un terme à la situation regrettable qui nous est signalée en favorisant la propagation exclusive de la langue française ».

13 Si ces établissements congréganistes furent des vecteurs d'introduction de la langue française en Tunisie, durant la période pré-coloniale, ils n'eurent pas d'influence directe sur la population tunisienne musulmane. F. Amoulet signale que seuls quatre élèves arabes suivaient les enseignements du collège Saint-Louis de Carthage en 1880. Cependant certains anciens élèves sortis de ces écoles devaient propager, ensuite, l'enseignement du français quand ils furent nommés professeurs à l'École militaire du Bardo ou au collège Sadaki de Tunis.

\section{Les enseignements nouveaux dans la Tunisie précoloniale}

En effet, le troisième tiers du XIX ${ }^{\mathrm{e}}$ siècle fut l'ère des réformes en Tunisie. Faisant partie de l'Empire ottoman, hommes d'État et intellectuels suivaient avec intérêt ce qui se passait à Istanbul où depuis 1839 les Tanzimat étaient appliquées en vue de réformer les institutions et de moderniser le pays. Comme leurs coreligionnaires d'Orient, les ulémas tunisiens étaient conscients du retard accusé par les pays musulmans par rapport aux pays européens d'Occident et ressentaient l'application de réformes nécessaires au monde musulman dont la Tunisie. Ils considéraient que l'un des facteurs du développement des pays musulmans était l'enseignement qui, selon eux, avait permis le développement technologique, militaire et économique des pays européens. Il fallait donc emprunter à ces pays ce qui fit leur force sans que cela pût porter atteinte aux fondements de la religion musulmane. 
Ce fut ainsi que, pour moderniser son armée, Ahmed Bey créa l'École polytechnique devenue École militaire du Bardo en 1840. Il fit appel, pour la diriger, à un officier d'origine piémontaise, arabisant ayant servi dans l'armée turque, le colonel Calligaris, remplacé, par la suite, par des officiers français. Celui-ci organisa les nouveaux enseignements où des matières profanes furent introduites et instaura l'enseignement des langues étrangères dont l'italien et le français, ces deux langues étant considérées comme des vecteurs d'introduction de la modernité en Tunisie : elles servaient, en effet, à l'acquisition de nouveaux savoirs et constituaient des moyens d'ouverture sur le monde extérieur. Les élèves de l'école étaient tenus, à la fin de leurs études, de traduire un ouvrage d'art militaire de l'une de ces langues en arabe, car l'enseignement classique en langue arabe était maintenu et enseigné par des cheikhs réformateurs de la Zitouna de Hinis, institution d'enseignement à dominante religieuse.

Des officiers sortis de l'école et leurs professeurs et leurs disciples de la Zitouna constituèrent le premier groupe de Réformistes qui tentèrent de mettre en pratique leurs idées nouvelles et leurs nouvelles conceptions de l'enseignement devenu, désormais, une « affaire de l'État » après qu'il fut l'affaire de la communauté.

17 L'arrivée de Kheredine au pouvoir, en 1873 en tant que Premier ministre, concrétisa cette nouvelle conception par la création d'une institution originale que fut le collège Sadiki de Tùnis qui ouvrit ses portes en 1875 ainsi que par des réformes successives de l'enseignement et des structures de la Zitouna de Tùnis.

18 La création du collège Sadiki répondait au dessein de son fondateur de favoriser la formation de fonctionnaires de l'État et de préparer aux fonctions libérales dont le pays pouvait avoir besoin pour son développement. Cette corrélation entre l'acquisition des savoirs et le développement était nettement évoquée par Kheredine dans son ouvrage Essai sur les réformes nécessaires aux États musulmans:

Je veux, écrit-il, réveiller le patriotisme des ulémas et des hommes d'État musulmans, et les engager à s'entraider dans le choix intelligent des moyens les plus efficaces pour améliorer l'état de la nation islamique, accroître et développer les éléments de sa civilisation, élargir le cercle des sciences et des connaissances, augmenter la richesse publique par le développement de l'agriculture, du commerce et de l'industrie, et pour établir, avant tout, comme base principale, un bon système de gouvernement d'où naît la confiance, qui produit à son tour la persévérance dans les efforts et le perfectionnement graduel en toutes choses, tel qu'il existe aujourd'hui en Europe.

19 Ainsi donc, élargir le cercle des connaissances et des sciences représentait, pour Kheredine, un objectif très important. En effet, sans elles, il ne serait guère possible pour un pays de se développer et d'échapper à la dépendance économique et partant politique vis-à-vis de l'Europe. Prenant comme prétexte la consommation de produits d'importation par certaines catégories sociales de Hinis, Kheredine stigmatisera ce phénomène en ces termes:

Il est facile de comprendre combien un pareil système de consommation est humiliant, antiéconomique et antipolitique. Humiliant parce que le besoin de recourir à l'étranger pour presque tous les objets de première nécessité démontre l'état arriéré des arts dans le pays ; antiéconomique, parce qu'il favorise l'industrie étrangère au détriment de l'industrie nationale, qui ne peut se livrer à la transformation des produits indigènes, transformation qui constitue une des principales sources de la richesse publique ; antipolitique surtout parce que la nécessité pour un État de recourir constamment à un autre est un obstacle à son indépendance et une cause de faiblesse, particulièrement si cette nécessité a 
rapport aux armes et au matériel de guerre ; car si on peut acheter ces choses en temps de paix, de gré à gré chez l'étranger, il est impossible de se les procurer à la même source en temps de guerre à $n$ 'importe quel prix. Nous ne possédons, dans notre état actuel, que des matières premières.

En effet, chez nous, l'éleveur de bétail, le cultivateur de coton et le céréaliculteur passent toute l'année dans des travaux pénibles, et finissent par vendre, à bas prix, leurs produits bruts aux Européens qui, dans un court délai, les leur revendent transformés par leur industrie à un prix dix fois plus élevé.

Cela n'a d'autre cause que la supériorité et le progrès de l'Europe dans les connaissances dont le développement est favorisé par des institutions politiques basées sur la justice et la liberté.

20 étrangères, notamment du français, de l'italien et du turc. L'utilisation de ces langues
était nécessaire pour l'acquisition de savoirs nouveaux lors du cursus scolaire au collège même mais aussi et surtout pour permettre aux élèves les plus méritants de poursuivre des études plus avancées dans des disciplines enseignées à l'étranger et en France, notamment. Le décret organique créant le collège Sadiki prévoyait, en effet, que les élèves les plus méritants seraient envoyés aux frais du collège poursuivre des études à l'étranger et une première délégation fut envoyée, en 1880, au lycée SaintLouis de Paris afin de préparer les concours d'entrée aux Grandes Écoles françaises. Mais l'envoi de ces missions fut suspendu dès l'instauration du Protectorat français, le gouvernement ayant préféré utiliser cette première promotion de Sadikiens en leur accordant des postes dans l'administration.

21 Ainsi la langue française, introduite volontairement par les Tunisiens avant la colonisation du pays en vue de favoriser son développement endogène servira l'administration coloniale et lui fournira les intermédiaires bilingues dont elle aura besoin pour la réalisation de sa propre politique.

\section{Le Protectorat et la langue française}

La nouvelle situation politique créé par l'occupation de la Tunisie influera sur le statut de la langue française. Instrumentalisée par le pouvoir colonial, elle aura une fonction idéologique et deviendra un facteur d'hégémonie.

Dans le premier cas, la langue française aura pour mission de favoriser la " conquête des cœurs et des esprits ", selon la formule consacrée. L'objectif du pouvoir colonial était, en effet, de franciser les populations autochtones et autres, habitant en Tunisie. Tels furent les objectifs présentés par Jules Ferry ${ }^{3}$, alors ministre de l'Instruction publique dans le cabinet Freyssinet, et Jules Jusserand, jeune fonctionnaire des Affaires étrangères ${ }^{4}$. Il semble que, dans un premier temps, les autorités coloniales n'avaient pas de projet cohérent d'instruction à fournir à la population tunisienne musulmane. Par contre, l'unanimité paraissait acquise pour préserver l'enseignement congréganiste, voire l'aider dans l'effort qu'il fournissait pour propager la langue française auprès de communautés européennes, notamment italienne et maltaise, que le nouveau pouvoir tentait de rapprocher de lui et d'assimiler à la population française minoritaire en nombre dans le pays. Le désir de francisation de la population tunisienne musulmane n'en était pas moins explicite tant chez Jules Ferry que chez Jules Jusserand. En effet, la note de J. Jusserand débute ainsi : 
Nous n'avons en ce moment de meilleur moyen de nous assimiler les Arabes de Tunisie, dans la mesure où cela est possible, que de leur apprendre notre langue, c'est l'avis de toutes les personnes qui les connaissent le mieux : nous ne pouvons pas compter sur la religion pour effectuer cette assimilation : ils ne se convertiront jamais au Christianisme ; mais à mesure qu'ils apprendront notre idiome une foule d'idées européennes se révéleront forcément à eux, l'expérience l'a suffisamment démontré. Dans la réorganisation de la Tunisie, une large part devra être faite à l'enseignement.

de l'Instruction publique en 1883. D instituera l'école franco-arabe où l'enseignement de la langue arabe sera maintenu mais où le français sera la langue dominante. Cette initiative recueillait l'appui et la sympathie des Tunisiens. Ainsi le cheïkh al-Islam, la plus haute personnalité religieuse du Royaume, s'adressait en ces termes à Louis Machuel :

Je tiens à ce que mes enfants apprennent la langue française, parce que de notre temps, on n'a pas le droit d'ignorer ce qui se passe dans les pays voisins et principalement en Europe, et votre langue est naturellement celle que nous devons étudier... Quant aux musulmans de Tunisie, ils s'habituent à votre présence et ne voient plus d'un mauvais œil les progrès que fait votre influence. Traitez-les avec justice ; respectez leurs croyances et leurs usages ; évitez de les froisser par des mesures arbitraires ou inopportunes; vous aurez vite achevé de les conquérir moralement ${ }^{5}$

Or ce nouveau statut donné à la langue française sera contesté aussi bien par les Prépondérants que par les nationalistes tunisiens. Il est vrai que les raisons de cette remise en question n'étaient pas les mêmes pour les deux parties. Le premier affrontement eut heu publiquement au congrès de l'Afrique du Nord de Paris en 1908 entre Khä̈rallah Ben Mustafa, membre du mouvement Jeunes-Tunisiens et Victor de Carnières, porte-parole du Parti colon. Pour cette première génération de Sadikiens, l'enseignement des langues étrangères et du français en particulier, était déjà inscrit au programme de leur institution et ne présentait, donc, à ce titre aucun danger pour eux. Ils n'avaient aucune appréhension, bien au contraire, à l'encontre de l'enseignement des langues étrangères et du français, particulièrement, introduit par leur idole, le Premier ministre Kheredine.

27 Nous avons vu les objectifs assignés à l'enseignement des langues étrangères durant la période pré-coloniale. Mais l'intérêt pour la langue va s'accroître durant la période coloniale car la langue française deviendra un facteur de promotion sociale. Elle sera la langue de l'administration et à ce titre sa connaissance deviendra obligatoire pour quiconque postulera à un poste dans l'administration publique ou le secteur privé. D'autre part, la langue française sera un instrument essentiel pour la poursuite d'études supérieures, en France notamment. Cette perception de la langue française ne pouvait que contrarier les tenants de la colonisation qui réclamaient pour les Tunisiens un enseignement rudimentaire. Victor de Carnières suggère de donner aux Tunisiens " un enseignement utile ». La Tunisie étant un pays agricole, l'enseignement à donner 
devrait former des ouvriers agricoles pour la colonisation. Il ajoute les griefs suivants contre l'école franco-arabe :

L'enfant indigène est malpropre.

L'enfant indigène est immoral.

L'enfant indigène qui ne sait pas un mot de français, en arrivant à l'école, entrave la marche de la classe.

Cette instruction fait de l'indigène le concurrent du Français.

Elle fait de l'indigène un quémandeur d'emplois qui, éconduit, se transforme en mécontent et en ennemi.

Cette instruction corrompt l'indigène, puisqu'il ne prend généralement que le mauvais côté de la civilisation.

Cette éducation rend l'indigène difficile à gouverner.

Cette conception n'était pas celle des Tunisiens. En effet, dès 1906 déjà, au congrès colonial de Marseille, Mohamed Lasram, seul délégué tunisien au Congrès, déclarait ceci :

... Les Musulmans ne sauraient parvenir au degré d'intellectualité des autres peuples et, par suite, ne pourraient prendre une part utile à l'activité économique de ces derniers s'ils devaient à tout jamais rester en dehors du mouvement scientifique dont se glorifient si justement les nations occidentales. D'où, Messieurs, cette conclusion naturelle qu'en Tunisie, l'enseignement des indigènes, pour être rationnellement conçu et préparer le rapprochement entre les deux races, doit nécessairement comprendre l'étude simultanée des langues française et arabe.

Après un examen critique de l'enseignement tunisien, il en vient aux propositions qu'il formule ainsi :

J'estime que pour accélérer le rapprochement des deux races, il faut :

$1^{\circ}$ - développer le plus largement possible l'enseignement des deux langues, française et arabe ;

2 admettre l'élément indigène à participer de manière plus active à la vie sociale européenne ;

$3^{\circ}$ - [...] préconiser, dans les différents milieux, la politique d'entente et d'association, à l'aide de publication en français et en arabe ayant respectivement pour objet de suggérer aux uns et aux autres une compréhension plus exacte de leurs droits et de leurs devoirs, comme aussi de leurs intérêts ;

$4^{\circ}$ - répandre les idées de mutuelle tolérance et de rapprochement dans les écoles françaises ;

$5^{\circ}$ - faciliter aux indigènes l'accès des établissements scolaires français, primaires et secondaires, pour qu'ils puissent se mêler, dès leur jeune âge à l'élément protecteur ;

$6^{\circ}$ - [...] utiliser plus largement qu'on ne l'a fait jusqu'ici, l'élite intellectuelle musulmane qui s'offre à servir de trait d'union entre les deux sociétés.

Nous percevons à travers ces deux fragments deux conceptions totalement différentes de l'école et de ses fonctions et des relations qui devraient régir les populations française et tunisienne dans la Régence. Si ces propositions tunisiennes peuvent répondre aux objectifs officiels du pouvoir du Protectorat, elles ne correspondent pas $\mathrm{du}$ tout aux vœux des Prépondérants qui continuent de condamner la politique d'association préconisée par Louis Machuel et soutenue par Paul Cambon.

Mais au-delà de la politique associationniste que les Tunisiens défendaient aux premiers temps de la colonisation et pour laquelle ils estimaient indispensable l'acquisition de la langue française, ils jugeaient, néanmoins, excessive la place occupée par cette même langue française au détriment de la langue arabe. En effet, la supériorité de la langue française était aussi manifeste dans l'enseignement primaire 
que dans l'enseignement secondaire. Dans celui-ci, l'enseignement en langue française variait de quinze heures et trente minutes à vingt-sept heures et trente minutes selon les cycles et les sections tandis que l'enseignement arabe variait de neuf heures à treize heures par semaine. Si l'enseignement en langue française recouvrait les mêmes disciplines que dans les sections modernes et classiques de renseignement français, l'enseignement en langue arabe touchait quant à lui la langue et la littérature arabes, l'histoire et la géographie du monde musulman, l'enseignement islamique, la traduction de textes administratifs, l'instruction civique, la calligraphie et la paléographie. S'il n'y avait pas d'hostilité à l'égard de la langue française, il y avait par contre contestation du rôle que le pouvoir colonial lui assigna par la suite. Elle demeurait, certes, un moyen d'acquisition de savoirs nouveaux mais elle devenait aussi un moyen d'affirmation de l'hégémonie coloniale. Elle avait pour objectif de franciser les populations autochtones et de créer une ségrégation sociale entre les Tunisiens qui possédaient la langue française et ceux qui l'ignoraient du fait même de la francisation de l'administration.

Mais cette ségrégation touchait également les fonctionnaires européens et les fonctionnaires tunisiens qui, possédant les mêmes diplômes, étaient traités différemment, les fonctionnaires européens ayant toujours les faveurs du pouvoir colonial.

On décèle à travers ces dispositions les craintes du pouvoir que les Tunisiens ne deviennent des citoyens à part entière et qu'ils ne remettent en cause l'hégémonie coloniale. C'était pour ces raisons que les postes de conception ne leur étaient pas accessibles, ni l'accès aux Grandes Écoles sous prétexte que les Tunisiens n'étaient pas des citoyens français. C'était aussi pour cela que l'enseignement supérieur leur était resté plus ou moins fermé puisque l'Institut des Hautes Études de Tunis ne fut ouvert qu'en 1945 et que le gouvernement français s'opposa à la création de facultés.

Ce ne sera qu'après la Deuxième Guerre que des réformes seront apportées au système scolaire tunisien et qu'un certain équilibre sera instauré entre la langue française et la langue arabe.

35 Ainsi, si au début du Protectorat l'objectif officiel était de franciser les populations autochtones et de favoriser, par l'utilisation de la langue française, l'entente entre celles-ci et la communauté française installée en Tunisie, une telle politique n'a pas recueilli l'assentiment des populations concernées.

En effet, pour les Prépondérants, l'enseignement devait servir à la formation de producteurs exclusivement, la langue française n'étant qu'un simple moyen de communication.

37 L'objectif du pouvoir, même s'il pouvait correspondre parfois à celui des Prépondérants, en différait, malgré tout, selon la conjoncture. Il devait tenir compte du rapport de force à maintenir entre lui et les populations autochtones mues, par ailleurs, par un sentiment nationaliste puissant, notamment après la Deuxième Guerre mondiale. Aussi devait-il développer la scolarisation et céder à certaines revendications dont la revalorisation de la langue arabe.

Mais, au-delà de la demande sociale, l'administration ne pouvait pas se passer des services d'agents intermédiaires compétents, bilingues. Aussi a-t-elle maintenu l'enseignement bilingue, réclamé également par les Tunisiens, et a-t-elle élargi l'enseignement sadikien à d'autres établissements secondaires, répondant ainsi à ses 
propres besoins et au succès que cet enseignement avait auprès des Tunisiens. C'est, d'ailleurs, de cet enseignement qu'est née la première génération de fonctionnaires tunisiens de l'État colonial français qui devinrent aussi les premiers contestataires du régime colonial.

Leur double culture et leur origine sociale ont été les facteurs d'homogénéisation de ce groupe qui constituera, désormais, une charnière entre la pensée occidentale, grâce à la langue française et la pensée arabe et tunisienne, grâce à leur culture en langue arabe.

Abdelwahab Bouhadiba voit dans ce phénomène une survivance du message de Kheredine. Il écrit ceci à ce propos :

En Tunisie le message de Kheredine et sa volonté d'un pluralisme culturel endogène vont se survivre à travers tout le Protectorat dans le collège Sadiki dont l'esprit marquera des générations entières, grâce à tout ce qu'il symbolise d'abord et grâce aussi à l'action inlassablement nationaliste mais ouverte et bilingue de l'Association des anciens élèves du collège Sadiki. Les efforts du Protectorat pour franciser le Collège trouveront une grande résistance et, pour l'essentiel, seront voués à l'échec. Si la Tunisie indépendante apparait comme davantage sensible aux séductions du pluralisme culturel, c'est peut-être aussi du fait que la culture arabo-musulmane classique n'y a pas été le seul champ dans lequel la volonté de survivre a pu trouver refuge. La culture sadikienne a été à la fois nationale et ouverte, orientée sans complexe vers le dedans et vers le dehors, affirmative du soi mais consciente en même temps que le dialogue interculturel $\mathrm{n}$ 'est pas forcément dépersonnalisant ${ }^{6}$.

41 Cette philosophie a continué de régir le comportement des responsables tunisiens, depuis l'indépendance de la Tunisie, qui ont rendu à la langue arabe la place qui devait être logiquement la sienne, sans négliger, pour autant, les langues étrangères et la langue française, plus particulièrement, qui reste, encore de nos jours, la deuxième langue de l'enseignement dans le pays.

\section{NOTES}

1. Cf. François Arnoulet, "La pénétration intellectuelle en Tunisie avant le Protectorat ", Revue Africaine, 1er et 2e trimestre 1954, p. 143. Nous reprenons à Louis Arnoulet l'essentiel de ce qui concerne l'enseignement européen durant la période pré-coloniale.

2. Mémoires et Documents. A.E.F., Tunis, vol. XI.

3. Copie d'une note autographe de M. Jules Ferry, A.A.E.F., Mémoires et Documents, vol. 10, 1.3.1882

4. Note sur l'instruction en Tunisie, A.A.E.F., Mémoires et Documents, Tunis, vol. XI.

5. Cité par Aimé Dupuy. La Tunisie dans les lettres d'expression française, Paris, Editions universitaires, 1956, p. 215.

6. Abdelwahab Bouhadiba, «Le pluralisme des cultures au Maghreb » in Culture et société, Tunis, Publications de l'Université, 1978. p. 258. 
INDEX

Mots-clés : Tunisie, Algérie, Maroc, Maghreb, colonisation, enseignement congréganiste, Protectorat français, Kheredine, collège Sadiki

Keywords : Tunisia, Algeria, Morocco, the Maghreb, colonization, congreganist teaching, French Protectorate, Kheredine, Sadiki school

\section{AUTEUR}

NOUREDDINE SRAÎEB

CNRS-IREMAM, Université de Provence 